\title{
Research on Servo System of Hydraulic Cylinder Test Bed
}

\author{
Yilun Han, Guangqing Ji, Pengcheng Qiu,Yangao Liang \\ Shandong University of Science and Technology, Qingdao 266590, China \\ jigqing@163.com
}

Key words: hydraulic cylinder; test-bed; PLC; servo system

\begin{abstract}
This paper through the study of the hydraulic cylinder test system, for the low-automation, low-automation, and inconvenient of old Hydraulic Cylinder Test bed, designed a set of hydraulic cylinder test bed system based on servo control. In the servo system the industrial computer as the upper machine, Siemens PLC as lower machine, touch screen as the auxiliary equipment. The proportional relief valve, electro-hydraulic servo valve and the sensor is applied to the servo system and the servo system used industrial maturity PID control algorithm. The the servo system is high precision, high degree of automation and meet production requirements.
\end{abstract}

\section{Introduction}

Hydraulic cylinder test and detection is an important means to guarantee the normal work of the hydraulic system[1]. Due to the traditional hydraulic cylinder test bed has the problem of low accuracy and low working efficiency, therefore, an accurate detection and intelligent hydraulic cylinder test-bed is the developing direction in the future[2]. The hydraulic cylinder test bed adopts the servo control system, using industrial computer as the upper machine, responsible for analog acquisition, data storage, etc.;using the Siemens PLC as lower machine, responsible for the control of the action logic of hydraulic components; also used touch screen as the auxiliary equipment. The servo system improved the system accuracy, automation and system stability.

\section{The servo system}

The hydraulic principle diagram of the servo control system is shown in fig. 1 . The servo system is composed of hydraulic pump, electro-hydraulic servo valve, proportional relief valve and pressure sensor and so on. The electro-hydraulic servo valve, proportional relief valve and pressure sensor formed pressure closed loop control. The pressure sensor detect the pressure value of the hydraulic cylinder port, and send the signal to PLC by A/D module. Then the PLC putout control signal after calculating. The signal is converted into voltage signal by $\mathrm{D} / \mathrm{A}$ module. In the end the voltage signal is amplified by the servo amplifier to control the opening of electro-hydraulic servo valve and proportional relief valve to control the pressure of the system. 


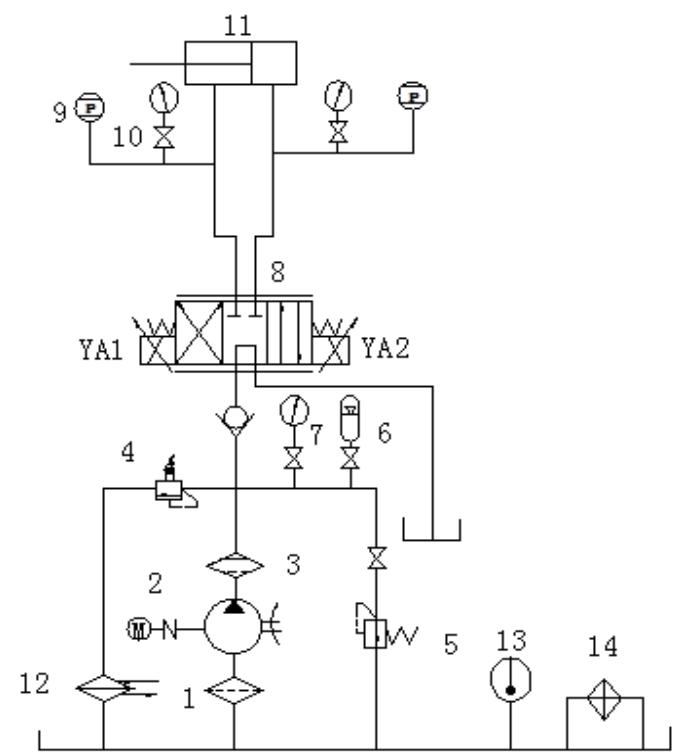

1.The coarse filter 2.Hydraulic pump power unit 3. Fine filter 4.Proportional relief valve 5.Safety valve Energy accumulator

7.Pressure gauge 8.Electro-hydraulic servo valve 9.pressure sensor 10. Globe valve 11 .Hydraulic cylinder 12 . The cooler

13. Thermometer 14.The heater

Fig.1 Hydraulic system diagram

\section{Control system hardware design}

\subsection{Control system structure design}

The control system is mainly composed of input switches, limit switches, pressure sensor, data acquisition, industrial computer, PLC controller, touch screen, switch output, analog output, as shown in Fig.2.

The system contains both digital and analog, data acquisition is divided into two parts, PLC is responsible for the collection of digital quantity such as switch button, limit switch etc., The data acquisition card is responsible for the collection of analog signal such as temperature and pressure sensor signal. Industrial computer is responsible for data processing, analysis, calculation, table storage and so on. The touch screen is mainly used for manual operation, parameter setting, test item selection and test curve display and so on. PLC as the core controller is responsible for all kinds of switch quantity input and output and analog output and controls the logical sequence of each component of the hydraulic system.

\subsection{PLC type selection and I/O distribution}

Control system selected S7-200 series PLC, according to the experimental requirements, the system has 13 switch input points and output points 6; analog input point 2 and the output point 2 . So choosed a PU224PLC with a PID controller and analog expansion module EM231 (4 input) and EM232 (2 output), and finally matched with a Touchscreen. this is most economical.

\section{Control system software design}

This system adopts Siemens, S7-200, the control program is divided into manual and automatic modes.Taking into account the scalability of the program, this paper uses the modular design method. According to the functional requirements of the hydraulic test bench is divided into the following modules: main program, Main program, parameter setting module, user module, data acquisition module, communication module, data storage module, operation module and test system module shown in Fig.3. 


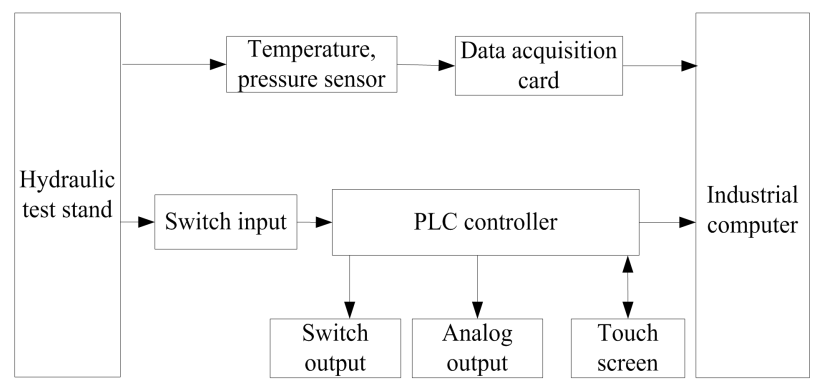

Fig.2 composition of the control system

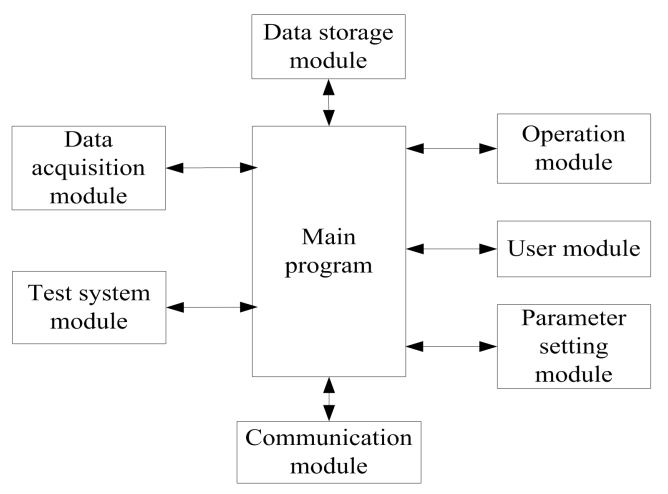

Fig.3 Function module structure diagram

\subsection{The PLC program design}

Open the system, enter the login page for authentication, if right enter the system or exit the system. then load the test parameters and ask for industrial computer to start the experiment . At the same time, the hydraulic system initialization, when the two meet began to test selection and test, test is completed choose whether or not to keep the test report, PLC program flow as shown in fig.4.

Introduce several main test automation program implementation process:

Trial run test. First hydraulic power units started, then 2YA energized ,the piston shift right;when the piston rod reaches the right end, right limit switch emit commutation signals, 1YA energized, the piston shift left; when the piston rod reaches the left end, left limit switch emit commutation signals, cycle more than five times, use PLC internal counter to count. Hydraulic cylinder should operate smoothly and can not have leakage[3]。

Start-up pressure test.First hydraulic power units started, then 2YA energized, the piston shift right; when the photoelectric switch detected the hydraulic cylinder piston rod, 1YA energized, the piston shift left, when the piston rod reaches the left end, left limit switch emit signals, the test is completed. The pressure sensor is used to record all changes in pressure and the maximum value is the starting pressure.

Durability test.First set the proportional relief valve pressure for the nominal pressure, then hydraulic power units started, 2YA energized, the piston shift right; when the piston rod reaches the right end, right limit switch emit commutation signals, 1YA energized, the piston shift left; when the piston rod reaches the left end, left limit switch emit commutation signals, cycle more than five thousand times.

\subsection{PID control algorithm}

PID control is a mature industrial control strategy[4][5].PID control system algorithm program diagram as shown in fig.5. First set a differential pressure $\triangle p$, then through the touch screen to set the test pressure of the system, The algorithm of PID control system is shown in figure 6 . First, set a differential pressure $\mathrm{P}$, then through the touch screen to preset system test pressure. When the hydraulic system work, the pressure sensor collect real-time signal send to PLC through the analog input module, the PLC controller processed the signal and get pressure $\mathrm{P}$ value. Then determine if $|p|>\triangle p$, execute proportional control in order to make the system pressure straight up (down) close to the target quickly, improve the response speed of system; if $|p|>\triangle p$, execute PID control in order to ensure accuracy of test pressure, satisfy the test requirement. 


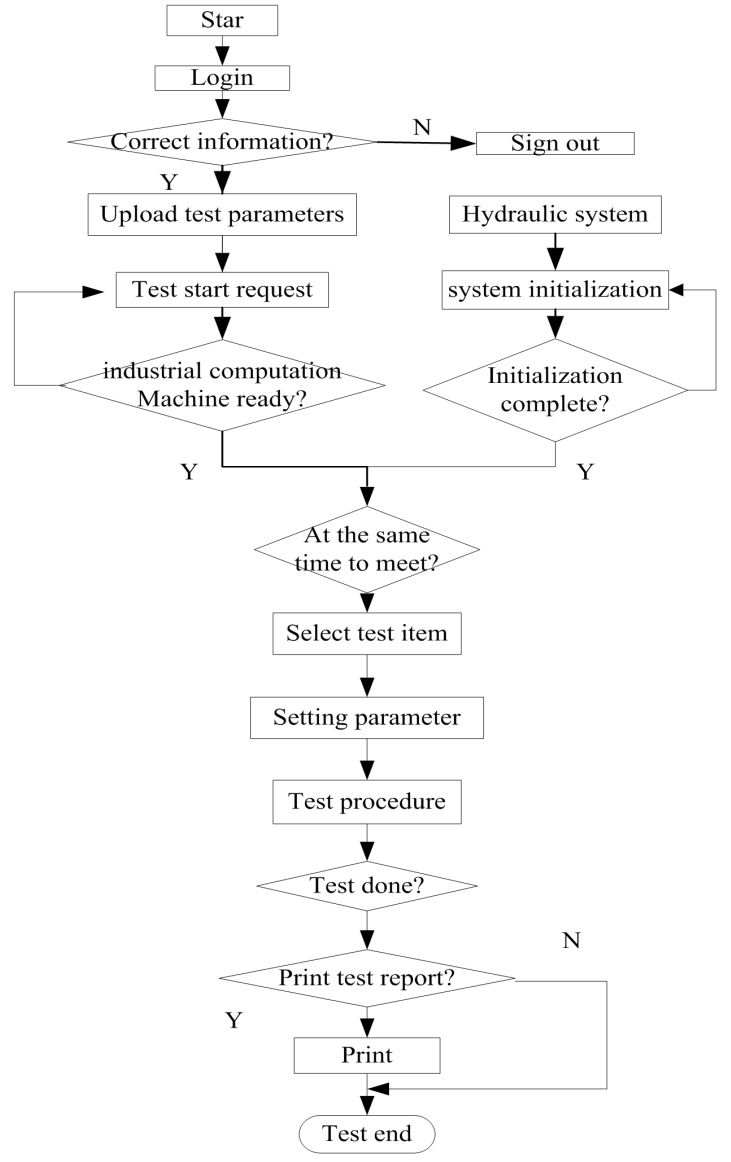

Fig.4 PLC program flow

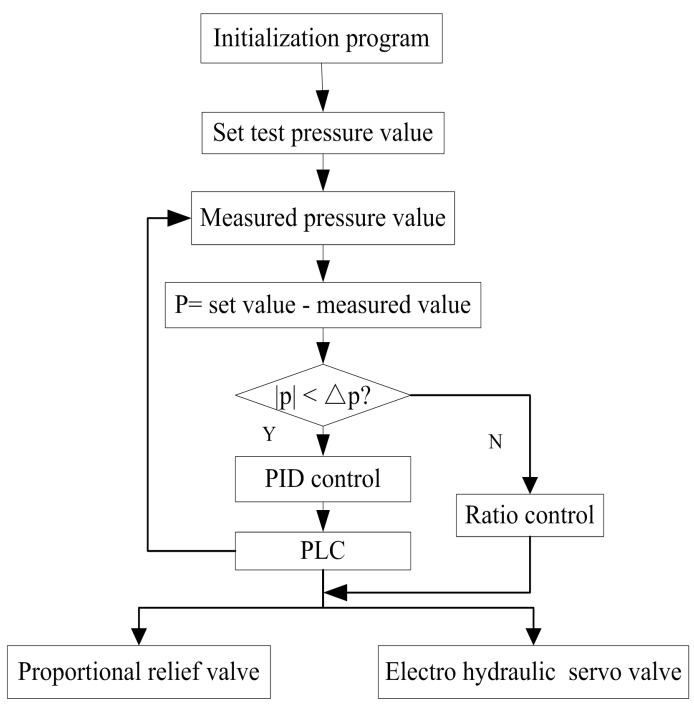

Fig.5 PID control system algorithm program diagram

\section{Conclusions}

This article mainly analyzed and designed the hydraulic servo control system and electric control system of the hydraulic cylinder test bed; using PID control algorithm, improved the precision of the system pressure and improved response speed of the system. The servo system improved the shortcomings of traditional hydraulic cylinder test platform which has low accuracy, low degree of automation and is inconvenient. The device can load or unload in any time, and it is easy to maintain, and it satisfies the producing requirements.

\section{Reference}

[1] Chen Dongning, Xu Haitao, Yao Chengyu. Test-bed Design and Engineering Practice for Large Diameter and Long Stroke Hydraulic Cylinder[J]. Machine Tool \& Hydraulics.2014(42): 79-84.

[2] Zhang Xiao-dong. The design of the computer control system of hydraulic cylinder test bed [J]. Mechanical Engineering and Automation, 2010 (1): 149-151.

[3] Wang Yi-qun, Gao Dian-rong. Hydraulic Engineer Technical Manual[M] Beijing:Chemical Industry Press, 2009.11.

[4] He Peng. Fuzzy PID controller design and simulation based on Matlab[J]. Microcomputer Applications,2010(26):59-64.

[5] Tao Yonghua. New type of PID control and its application[M]Beijing:Mechanical industry press, 2002.9 